\title{
Basophils and mast cells in immunity and inflammation
}

\author{
Yoshinori Yamanishi $^{1} \cdot$ Hajime Karasuyama ${ }^{1}$
}

Received: 30 June 2016 / Accepted: 5 July 2016 / Published online: 12 July 2016

(C) Springer-Verlag Berlin Heidelberg 2016

Basophils and mast cells were originally described, by Paul Ehrlich more than 130 years ago, as characteristic cells showing metachromatic stain with basic aniline dyes. They were long thought to be associated with allergic inflammation and parasitic infection, because their accumulation was frequently observed at inflamed sites of these diseases. However, the functional significance of these cells in vivo, particularly basophils, remained ill-defined compared with other immune cells such as T and B cells.

Basophils and mast cells share common phenotypic features, including the presence of preformed metachromatic granules and the surface expression of the high-affinity receptor for $\operatorname{IgE}(\mathrm{Fc \varepsilon RI})[1,2]$. They function as effector cells that produce a common set of bioactive molecules such as histamine, cytokines, chemokines, lipid mediators, and proteases in response to IgE plus antigens. These mediators elicit inflammatory immune responses, including allergic inflammation and protective reaction against parasitic infections. Nonetheless, basophils and mast cells exhibit fundamental differences in their anatomical localization, life span, and ability to proliferate. Basophils are the rarest blood granulocytes with a short life span (less than 3 days). They mature into terminally differentiated cells in the bone marrow, and circulate in the peripheral blood unless inflammation occurs. Basophils do not usually proliferate after maturation [3]. In

This is the introduction to Basophils and Mast Cells in Immunity and Inflammation - Dr Hajime Karasuyama

Yoshinori Yamanishi

yamanishi.mbch@tmd.ac.jp

1 Department of Immune Regulation, Graduate School of Medical and Dental Sciences, Tokyo Medical and Dental University,

Tokyo 113-8519, Japan contrast, mast cells are long-lived (up to months) tissueresident cells that are enriched around blood vessels. They fully differentiate within peripheral tissues, but still keep the ability to proliferate and self-renew $[4,5]$. Thus, it is now accepted that basophils and mast cells are distinct cell types with certain overlapping effector functions to elicit host protective or allergic responses [6]. Beyond these effecter functions, recent studies have illustrated the immunoregulatory roles of basophils and mast cells in health and disease. Notably, the recent development of analytical tools, including genetically engineered mice in which either basophils or mast cells can be selectively ablated, has made it possible to clearly identify the distinct and non-redundant roles of basophils and mast cells in the immune system. The present issue of Seminars in Immunopathology, entitled "Basophils and Mast Cells in Immunity and Inflammation", focuses on the recent advances in our understanding of the biology and pathology of basophils and mast cells, including their ontology and roles in allergic inflammation, protective response against parasitic infections and immune regulation.

Huang et al. [7] give an overview of the transcriptional regulation for lineage commitment of basophils and mast cells. They focus on recent advances in the understanding of origin and molecular regulation of basophil and mast cell development, in which the transcription factors $\mathrm{C} / \mathrm{EBP} \alpha$ and MITF are critical for specifying basophil and mast cell fate, respectively, in a directly antagonistic fashion.

Oetjen et al. [8] provide new insights into basophil heterogeneity. Basophils have a phenotypic heterogeneity and exhibit distinct features for activation under different cytokine milieu. Thymic stromal lymphopoietin (TSLP), in addition to IL-3, has recently been identified as a key regulator of basophil development. TSLP-elicited basophils show higher expression of receptors for IL-18 and IL-33 along with lower responsiveness to $\mathrm{IgE}$ plus antigens, so that they produce 
higher levels of IL-4 in response to these cytokines than do IL3 -elicited conventional basophils. Thus, TSLP-elicited basophils appear to play a critical role in the IgE-independent innate immune responses.

Otsuka et al. [9] discuss the roles of basophils and mast cells in cutaneous inflammation. Analysis of mice with diphtheria toxin-based genetic depletion of either basophils or mast cells has revealed that basophils are responsible for cutaneous Th2 skewing to haptens and peptide antigens but not protein antigens, while mast cells promote the development of contact hypersensitivity via activation of dendritic cells in the skin. Thus, both basophils and mast cells play important roles in cutaneous allergic inflammation.

Takeda et al. [10] review the regulation of allergic inflammation by E-NPP3 (CD203c) expressed on basophils and mast cells. E-NPP3 functions as a nucleotide-converting ectoenzyme and inhibits ATP-dependent immune responses by hydrolyzing ATP. Basophils and mast cells release ATP upon FceRI cross-linking, and the resultant ATP activates these cells in an autocrine manner. E-NPP is rapidly induced on basophils and mast cells, and consequently hydrolyzes extracellular ATP to prevent ATP-dependent excess activation of these cells. Thus, E-NPP3 plays an important role in the regulation of basophil- and mast cell-mediated allergic responses.

Mukai et al. [11] focus on the roles of mast cells in host defense against parasites and venoms. They have reviewed evidence that, in some settings, mast cells and IgE can contribute to host defense against parasites, particularly helminths. In other settings, however, mast cells and IgE may have net effects that contribute to the pathology during such infections and that favor the survival or fecundity of the parasite. In addition, mast cells and $\mathrm{IgE}$ also can play prominent roles in innate and adaptive immune responses to venoms.

Eberle et al. [12] discuss the role of basophils in protective immunity to parasitic infections. They review the current knowledge regarding the protective and immunomodulatory roles of basophils during infection with a variety of endo- and ectoparasites. Basophils can be activated either directly by parasite-derived factors or indirectly by recognition of parasite-derived antigens via IgE. In response to IgE plus antigens, basophils exert their ability to protect the host from helminthic infection through secretion of IL-4 and IL-13, which in turn activates other cell types including goblet cells to mediate worm expulsion. IgE-mediated basophil activation is also important for protection against the secondary tick infection.

Yamanishi et al. [13] focus on the versatile roles of basophil-derived IL-4 in immunity. Basophils can rapidly produce large quantities of IL-4 in response to a variety of stimuli such as IgE plus antigens, cytokines, TLR ligands, and allergenic proteases. Basophil-derived IL-4 has been shown to regulate other types of cells, including T cells, B cells, group 2 innate lymphoid cells (ILC2s), macrophages, fibroblasts, and endothelial cells. These stimulated cells subsequently contribute to the positive and negative regulation of a variety of immune responses in health, in diseases such as parasitic and bacterial infections, allergy, and autoimmune diseases, and in tissue homeostasis.

Finally, Morita et al. [14] discuss the regulatory roles of mast cells in immune responses. They review the current knowledge of the mechanisms by which mast cells suppress immunological disorders, including skin allograft rejection and graft-versus-host diseases, contact hypersensitivities, and innate-type allergic airway inflammation. During the papain-induced innate-type airway inflammation, epithelial cell-derived IL-33 stimulates mast cells to produce IL-2, which induces the activation of regulatory T cells (Tregs). Activated Tregs release IL-10 that inhibits the proliferation and Th2 cytokine production of ILC2s, resulting in the suppression of ILC2-mediated airway inflammation. Thus, mast cells play a regulatory role in the papaininduced innate-type airway inflammation.

In conclusion, the present issue of Seminars in Immuno pathology highlights the development of basophils and mast cells and their detrimental and beneficial roles under physiologic and pathologic conditions in the immune system. Although we need to address more detailed mechanisms by which basophils and mast cells exert their functions, it is now clear that basophils and mast cells play crucial and non-redundant roles in allergy, protection against parasitic infections, and other types of immunological disorders. We hope these reviews will contribute to better understanding of the biological significance of basophils and mast cells in immunity. We greatly appreciate the authors' valuable contributions.

\section{References}

1. Galli SJ (2000) Mast cells and basophils. Curr Opin Hematol 7:32-39

2. Stone KD, Prussin C, Metcalfe DD (2010) IgE, mast cells, basophils, and eosinophils. J Allergy Clin Immunol 125:S73-S80

3. Karasuyama H, Mukai K, Obata K, Tsujimura Y, Wada T (2011) Nonredundant roles of basophils in immunity. Annu Rev Immunol 29:45-69

4. Galli SJ (1993) New concepts about the mast cell. N Engl J Med 328:257-265

5. Metcalfe DD, Baram D, Mekori YA (1997) Mast cells. Physiol Rev 77:1033-1079

6. Voehringer D (2013) Protective and pathological roles of mast cells and basophils. Nat Rev Immunol 13:362-375

7. Huang H, Li Y, Liu B (2016) Transcriptional regulation of mast cell and basophil lineage commitment. Semin Immunopathol. doi:10.1007/s00281-016-0562-4

8. Oetjen LK, Noti M, Kim BS (2016) New insights into basophil heterogeneity. Semin Immunopathol. doi:10.1007/s00281-016-0567-Z

9. Otsuka A, Nonomura Y, Kabashima K (2016) Roles of basophils and mast cells in cutaneous inflammation. Semin Immunopathol. doi:10.1007/s00281-016-0570-4

10. Takeda K, Tsai SH (2016) Regulation of allergic inflammation by the ectoenzyme E-NPP3 (CD203c) on basophils and mast cells. Semin Immunopathol. doi:10.1007/s00281-016-0564-2 
11. Mukai K, Tsai M, Starkl P, Marichal T, Galli SJ (2016) IgE and mast cells in host defense against parasites and venoms. Semin Immunopathol. doi:10.1007/s00281-016-0565-1

12. Eberle JU, Voehringer D (2016) Role of basophils in protective immunity to parasitic infections. Semin Immunopathol. doi:10.1007/s00281-016-0563-3
13. Yamanishi Y, Karasuyama H (2016) Basophil-derived IL-4 plays versatile roles in immunity. Semin Immunopathol. doi:10.1007 /s00281-016-0568-y

14. Morita H, Saito H, Matsumoto K, Nakae S (2016) Regulatory roles of mast cells in immune responses. Semin Immunopathol. doi:10.1007/s00281-016-0566-0 